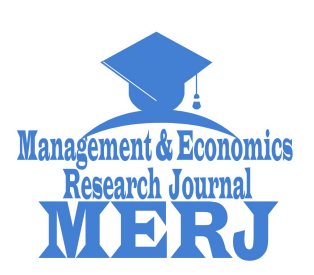

https://doi.org/10.48100/merj.v1i2.35

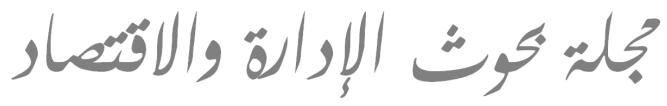

Management \& Economics Research Journal Vol. 1 No. 2 (2019): June المجلد 1 العدد 2 (2019): جوان

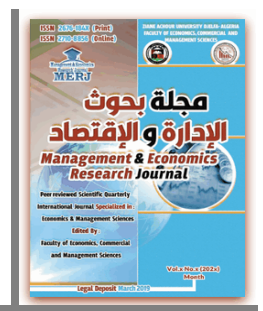

\title{
Deficit Budgetaire Et Financement Non Conventionnel En Algerie
}

\section{Budget Deficit and Unconventional Financing in Algeria}

\author{
Hadjer OULD HENNIA ${ }^{1}$ \\ ${ }^{1} \mathrm{PhD}$, Monetary and Financial Economics, Oran 2 University (Algerie) \\ e-mail : hadjer.ouldhennia@gmail.com
}

Reçu: 12-05-2019

Accepté: 26-06-2019

Publié: 30-06-2019

\section{Résumé:}

L'objet de cette étude est d'analyser la relation entre le déficit budgétaire et le financement non conventionnel en Algérie. Les résultats de notre analyse indiquent que la chute des prix du pétrole à la mi-2014, entraînant une baisse des recettes pétrolières et gazières, qui est la principale source de financement de l'économie, a provoqué des déficits budgétaires, suite à cela les responsables ont essayé de trouver de nouvel instrument de financement alternatif. Donc face à cette crise financière, le gouvernement a décidé de recourir au financement non conventionnel interne.

Mots clés: Déficit budgétaire, Planche à billet, Algérie.

Classification Jel: $\mathrm{H} 61, \mathrm{H} 62, \mathrm{H} 72$.

Abstract:

The purpose of this study is to analyze the relationship between the budget deficit and unconventional financing in Algeria. The results of our analysis indicate that the drop in oil prices in mid-2014, resulting in lower oil and gas revenues, which is the primary source of financing for the economy, has led to budget deficits, following which officials have tried to find a new alternative financing instrument. So in the face of this financial crisis, the government decided to resort to unconventional internal financing.

Keywords: Budget Deficit, Billboard, Algeria.

JEL Classification: H61, H62, H72.

\section{Introduction :}

-Auteur correspondant : Dr. Hadjer OULD HENNIA, e-mail : hadjer.ouldhennia@gmail.com 
La politique budgétaire est la stratégie que met en œuvre un gouvernement pour agir sur l'économie du pays en utilisant son pouvoir de fixer les recettes de l'Etat et les priorités dans la répartition des dépenses publiques.

L'effet principal du budget se situe au niveau de la demande, qu'il s'agisse de l'importance et de la nature des dépenses, des recettes et du déficit ou l'excédent. Mais il influe aussi sur l'offre et sur les circuits de financement (Dictionnaire d'économie et des sciences sociales, 1992).

La politique budgétaire nationale demeure le principal instrument pour faire face à des chocs économiques ponctuels, car les gouvernements nationaux ne peuvent pas recourir à la politique monétaire pour influencer les variations de la conjoncture économique. La politique budgétaire comprend deux postes : les recettes publiques et les dépenses publiques sont financées par: les recettes fiscales, non fiscales, les dons et les prêts. Pour la collecte des ressources, l'Etat accorde une importance particulière aux normes fiscales, à une gestion rationnelles des dépenses publiques.

L'Algérie, ces trois dernières années a enregistré de faibles recettes, suite à la chute libre des prix du baril, où elle se trouvé dans une situation de crise de ses finances publics.

Afin de faire face à ce déficit budgétaire, un nouvel instrument a été introduit qui est «le crédit non conventionnel », ce travail vient répondre aux questions suivantes:

Qu'est ce qu'un déficit budgétaire et comment a été financer en Algérie

\section{Cadre conceptuel}

Cette partie, revue de littérature s'attachera à présenter les concepts de la politique budgétaire ainsi que les modes de financement du déficit du trésor.

\subsection{Le trésor public:}

2.1.1 Définition : c'est un établissement financier non doté de la personnalité juridique ou morale. Le trésor public administrativement est rattaché au ministère des finances, ses fonctions et ses taches sont d'ordres financiers, économiques et comptables.

Les fonctions financières ont pour objet de garantir l'équilibre financier pour subvenir aux besoins financiers de l'Etat et dans ce cadre le trésor public est chargé de :

- Gérer l'équilibre de la trésorerie, par le biais des dépôts de fonds des administrations et collectivités publique ainsi que les déports de fonds des particuliers.

- Gérer les ressources financières de l'Etat. Les fonctions économiques du trésor public servent comme un outil a la politique économique a travers une extension de la politique monétaire et dans ce cadre il jouit le rôle de banquier de l'Etat et participe au financement de l'Etat.

\subsubsection{Rôle du trésor public: (SADDIKI, Décembre 2014)}

I. Exécution des dépenses publiques et recouvrement des recettes En matière d'exécution des dépenses publiques et recouvrement des recettes, le trésor public est chargé de la mission de contrôle sur l'exécution des budgets public en tant qu'organe de contrôle préalable. 
Les opérations de contrôle s'applique dans l'exécution des budgets de l'Etat, des institutions et administrations publiques, des collectivités locales et des établissements publics a caractère administratif.

II. Banquier de l'Etat : Le rôle banquier du trésor en effet étendu de façon considérable pour le développement des opérations financières qui concernent le secteur public et privé. Par le canal de ses correspondants le trésor public manié les fonds d'une clientèle diversifiées et dont il a le rôle d'une banque de dépôt, et également il joue ce rôle sur le marché des capitaux.

III. Caissier de l'Etat : Il gère le compte de l'Etat auprès de la banque d'Algérie ; principe de l'unité de caisse, il a assure le recouvrement de toutes les recettes publiques et paie les dépenses ordonnancées sur les budgets publics.

La politique budgétaire et la politique monétaire constituent les principaux leviers de la politique économique.

\subsection{La politique budgétaire :}

La politique budgétaire est l'ensemble des mesures ayant des conséquences sur les ressources ou les dépenses inscrites au budget de l'Etat et visant directement à agir sur la conjoncture (Gerard THORIS, 2000, p. 11).

Par politique budgétaire, nous entendons une politique mise en œuvre par le gouvernement pour agir sur l'économie du pays en utilisant son pouvoir de fixer les recettes de l'État et les priorités dans la répartition des dépenses publiques. Elle recouvre ainsi l'utilisation des dépenses et des recettes des administrations publiques par le gouvernement pour réguler le rythme de l'activité économique afin d'atteindre ses objectifs que ce soit dans le domaine social ou économique (TOUREV. P, 2015).

Cette politique consiste à utiliser certains instruments tels que les dépenses publiques, l'endettement public et les prélèvements fiscaux pour influer sur la conjoncture économique. Elle s'appuie sur l'élaboration du budget de l'Etat qui n'est autre que la prévision de l'ensemble des recettes et dépenses de l'Etat pour l'année. De cette présentation succincte de la politique budgétaire, il ressort deux notions principales et importantes, que sont les recettes et les dépenses publiques.

La règle veut que les recettes et les dépenses s'égalisent afin que le budget soit équilibré, un excédent ou un déficit selon les courants de pensée est applaudi ou décrié. Ces différentes conceptions ont donné naissance à une politique budgétaire qui utilise le solde budgétaire comme un instrument de politique économique en ce sens qu'il donne un reflet de l'activité économique.

Jusqu'à la crise de 1930, la gestion des finances publiques a eu pour principal objectif d'assurer le financement des services publics. Le volume des dépenses de l'Etat n'était considéré comme une variable susceptible d'influencer le niveau d'activité de l'économie. L'analyse de l'économiste britannique J. M. KEYNES a modifié cette conception en soulignant l'impact de la politique budgétaire sur le niveau d'activité économique d'un pays. 
Les recettes publiques proviennent de la politique fiscale. La fonction de la fiscalité est d'assurer le financement de la production des services traditionnels. Elle sert également à la redistribution du revenu. Elle est utilisée pour réduire les disparités sociales grâce au recours à la progressivité des taux d'imposition, à divers abattements et déductions pour les charges personnelles et familiales. Ainsi, l'Etat doit utiliser son pouvoir de taxation, de dépense et d'endettement pour réaliser une allocation des ressources.

Les recettes publiques financent les dépenses de l'Etat, qui sont les dépenses du secteur public engagées en vue d'un intérêt public. Les dépenses publiques sont les dépenses effectuées par l'Etat pour son fonctionnement et celles orientées vers les secteurs prioritaires (sociaux, sécurité) pour l'amélioration du bien-être des populations.

De JANVRY et al. (1991), D et L. DEMERY (1992), LIPTON et RAVALTON (1993) et AHO et al. (1997) ont tous montré l'impact social des dépenses publiques. Ils ont montré que les dépenses publiques permettent d'accroître la productivité et les profits des facteurs et par conséquent d'augmenter les revenus des ménages. Ainsi, nous pouvons dire que la dépense publique dépend de la politique budgétaire (De JANVRY, 2017).

\subsection{Le budget :}

C'est l'un des mots qui a avoir traversé deux fois la manche. Signifiant au moyen âge, la bougette désignait «le porte monnaie »; après, les Anglais l'ont repris et l'ont transformé en «budget », en 1789, et pendant la révolution française, les français l'ont repris.

Le budget de l'Etat est une loi (la loi de finance) votée chaque année et autorisant l'Etat à prélever certaines ressources (impôts et taxes) et à dépenser ces ressources d'une manière prévue précisément par la loi (MATTHIEU, 1998).

Alors, le budget est un élément comptable comportant l'ensemble des recettes et des dépenses prévisionnelles d'un agent économique (un ménage, l'Etat...) au cours d'un exercice comptable

2.3.1 Le budget révisé : les prévisions doivent être révisées au cours de l'exercice comptable si on constate que le budget prévisionnel n'est pas conforme aux réalisations.

Le budget est inutile si y'aura pas de suivi s'assurant de son respect, et sans mesures correctrices. Ces mesures peuvent porter sur le budget lui-même, sur le fonctionnement de l'agent économique, ou sur l'enregistrement comptable.

Signalons que le budget bien qu'il soit, par définition un état comptable de prévision de dépenses ou de recettes, nous constatons dans tous les textes législatifs l'emploi systématique de l'expression budget prévisionnel.

\subsection{Les recettes et les dépenses du budget de l'Etat :}

Le budget englobe les dépenses et les recettes annuelles de l'Etat, il sert comme instrument pour agir sur la conjoncture. 


\subsubsection{Les recettes de l'Etat :}

L'Etat a énormément besoin de ressources afin de couvrir ses dépenses multiples, ces ressources constituent l'argent de l'Etat. Il existe une variété de moyens pour qu'il se procure cet argent :

- Fiscalités pétrolière,

- Les recettes fiscales tirées de l'impôt ;

$\checkmark$ impôt sur le capital,

$\checkmark$ impôtsur le revenu,

$\checkmark$ et impôt sur la dépense.

- Les ressources non fiscales;

$\checkmark$ Les taxes administratives;

$\checkmark$ Les ressources domaniales;

$\checkmark$ Les ressources parafiscales;

- Contribution au budget de l'Etat,

- Et les ressources du trésor provenant du financement monétaire (moyen de trésorerie);

$\checkmark$ les emprunts publics;

$\checkmark$ les capitaux extérieurs et ;

$\checkmark$ le financement monétaire.

Pour financer ses dépenses publiques, l'Etat peut en plus des ressources déjà cités en haut recourir à ces trois autres sources des revenus « extra fiscaux», mais il ne recourt à ces moyens que dans le cas d'une impasse budgétaire (décaissement supérieur aux encaissements). L'importance de ces méthodes varie suivant la conjoncture et la structure économique, et leurs effets sont très différents au regard des objectifs assignés à la politique des finances publiques.

\subsubsection{Les dépenses de l'Etat :}

Les dépenses sont impératives et obligatoires, ils comprennent les dépenses de fonctionnement, les transferts et les dépenses en capital...etc., ils représentent les consommations des revenus par l'Etat.

Ce dernier établie ses recettes en fonction de ses dépenses et non ses dépenses en fonction de ses recettes où les besoins de l'Etat sont financés par les citoyens à travers les charges que ceux-ci doivent supporter.

Nous pouvons classés les dépenses de l'Etat sous deux catégories:

- les dépenses courantes

$\checkmark$ les dépenses de fonctionnement,

$\checkmark$ le service de la dette publique (intérêt à verser aux créanciers de l'Etat),

$\checkmark$ les subventions et transferts courants.

- les dépenses en capital : 


\section{les investissements directs \\ Les investissements indirects.}

\subsubsection{Le déficit du budget :}

C'est la situation budgétaire d'un Etat souverain qui voit les dépenses publiques annuelles dépasser les recettes publiques annuelles. Le cumul des déficits budgétaires constitue le stock de dette publique: c'est donc la somme des déficits budgétaires annuels qui forme la dette souveraine (Lexique boursier).

Le déficit budgétaire est, pour une année donnée, le solde négatif du budget d'un Etat lorsque ses dépenses (hors remboursement d'emprunt) excèdent ses recettes (hors emprunt). L'Etat ne pouvant être en cessation de paiement, un déficit budgétaire nécessite que celui-ci contracte de nouveaux emprunts qui ont pour conséquence d'augmenter la dette publique (TOPICTIONNAIRE).

Donc, on ne peut parler de déficit budgétaire « un solde négatif» que dans la situation où les recettes de l'Etat (Impôts et charges sociales) sont inférieures à ses dépenses (administration, éducation, dépenses sociales...) au cours d'une année ou bien les dépenses publiques pour une année sont supérieures aux recettes publiques, tout cela est exprimé par l'insuffisance des recettes où «l'Etat dépense d'abord ».

\subsection{Les financements du déficit :}

Les dépenses et les recettes ne s'égalisent pratiquement pas. En règle générale, l'Etat ordonne des dépenses (emplois) qui ont toujours été supérieures aux ressources dont il dispose.

Alors, tout agent économique «Etat,... », Dépensant plus que ses recettes doit trouver une source de financement pour combler son déficit budgétaire.celà peut se faire soit, par voie d'endettement c'est-à-dire suite a un emprunt auprès des agents qui ont un excédent «l'épargne», ou bien suivant l'approche de «la création monétaire».

Afin que les comptes soient équilibrés, le déficit peut être financé par plusieurs méthodes :

- Les réserves préalablement accumulées à partir d'excédents budgétaires réalisés les années antérieures ;

- la hausse des impôts et taxes ;

- l'emprunt;

- ou bien l'émission monétaire « la planche à billet».

«Le crédit non conventionnel » faisant l'objet du présent colloque, sur lequel doit être mise l'accent, est appelée aussi « la Planche à billet » ou bien la création de la monnaie.

Donc, l'Etat peut emprunter auprès de créanciers privés (emprunt qui crée de la dette) ou auprès de la banque centrale en ayant recours à la création monétaire (la fameuse planche à billets qui crée de l'inflation) cela veut dire qu'il émet de la monnaie en fonction de la demande du trésor.

Le financement monétaire (ou monétisation de dette) consiste pour une banque centrale à financer directement le budget du gouvernement central. Ce processus est parfois 
appelé péjorativement la « Planche à billets ». Elle fait référence aux processus de production de monnaie physique, mais en pratique il s'agit aujourd'hui plus d'écriture comptable.

Pour financer son déficit budgétaire, l'État peut aussi recourir au financement monétaire, c'est à dire à la création monétaire. Dans ce cas, la Banque centrale consent une avance à l'État en créditant le compte du Trésor public ce qui provoque l'accroissement de la masse monétaire.

Ce mode de financement du déficit budgétaire est un outil économique puissant, qui peut contribuer à stimuler l'économie, cependant il est risqué dans le cas où la masse monétaire s'accroit plus rapidement que la production, ce qui pousse le rythme de l'inflation à s'accélérer.

\section{3. le financement non conventionnel en Algérie :}

L'Algérie souffre d'une crise financière qui dure depuis 3 ans, suite à une libre chute des prix du pétrole, ce qui l'a poussé à introduire ce nouvel instrument afin de financer le déficit budgétaire.

Le nouvelle loi (Loi n 17-10, 2003) portant amendement de l'ordonnance relative à la monnaie et au crédit modifie et complète l'ordonnance de 2003, en introduisant un article 45bis qui stipule que «la banque d'Algérie procède, dés l'entrée en vigueur de la présente disposition, à titre exceptionnel et durant une période de cinq (5) années, à l'achat directement auprès du Trésor, de titres émis par celui-ci, à l'effet de participer, notamment :

- à la couverture des besoins de financement du Trésor ;

- au financement de la dette publique interne ;

- au financement du Fonds National d'Investissement (FNI).

en outre, l'article 45bis note que « Ce dispositif est mis en œuvre pour accompagner la réalisation d'un programme de réformes structurelles économiques et budgétaires devant aboutir, au plus tard, à l'issue de la période susvisée, notamment, au rétablissement :

- des équilibres de la trésorerie de l'Etat ;

- de l'équilibre de la balance des paiements. »

Pour rappel, les pouvoirs publics avaient annoncé les montants (Abderrahmane RAOUIA, 2017) qu'ils prévoyaient de tirer pour financer le déficit du Trésor, à savoir 570 milliards de dinars pour boucler l'exercice 2017, 1815 milliards de dinars pour celui en cours et enfin 580 milliards pour 2019.

Alors, en vue d'introduire ce nouvel instrument, le Gouvernement a élaboré ce projet de loi portant amendement de la loi sur la monnaie et le crédit dont l'objectif est d'autoriser la Banque d'Algérie, à titre exceptionnel et durant une période de cinq années, à procéder à l'achat direct de titres émis par le Trésor.

Il permettra de couvrir les besoins de financement du Trésor :

- de financer le remboursement de la dette publique interne,

- les titres de l'Emprunt national pour la croissance levé en 2016, 
- les titres du Trésor émis en contrepartie du rachat de la dette bancaire de Sonelgaz

- et les titres du Trésor émis au profit de la Sonatrach en compensation du différentiel sur les prix des carburants importés et de l'eau dessalée ;

- de permettre au Trésor de doter en ressources le Fonds National d'Investissement (FNI), au titre des prises de participation de l'Etat dans des investissements ou de financements à long terme de programmes publics d'investissements.

Cet instrument non conventionnel dont l'objet est de permettre la mobilisation par le Trésor de financement exceptionnel revêt un caractère transitoire, limité dans le temps à cinq années.

Le recours à cet instrument est accompagné par le programme de réformes structurelles économiques et budgétaires suivant :

- la rationalisation de la dépense publique,

- l'optimisation des recettes fiscales

en d'autre termes, recourir à la planche à billet et tenter de réduire les dépenses d'un Etat qui n'a pas pu équilibrer son budget, donc cela doit être respecter pour aboutir, au plus tard à l'issue de la période considérée, a restaurer des équilibres macroéconomiques et financiers (les équilibres de la Trésorerie de l'Etat et l'équilibre de la balance des paiements).

Nous pensons que le recours à la planche à billet en Algérie élaborer afin de combler le déficit budgétaire, et qui ne sera pas destiner à la consommation, selon les responsable, mais plutôt vers le budget d'investissement exclusivement afin de relancer la machine économique du pays, conduira certainement à l'explosion des prix, aussi l'inflation sera alimentée par la consommation, vu qu'en Algérie, la production est encouragée mais la quasi-totalité des entreprises ne sont pas exportatrices, cela implique que cette production sera destinée au marché interne.

Cette monétisation de dette mènera à des conséquences néfaste sur l'ensemble des agrégats macroéconomique, tel que, la dévaluation du dinar et une perte du pouvoir d'achat des algériens qui sont déjà affectés par une dépréciation continue du dinar, comme le souligne l'économiste CHRISTOFER DEMBIK - Responsable de la recherche macroéconomique chez Saxo Bank, Paris - « ce processus n'est ni plus ni moins qu'un recours à la 'planche à billets', c'est une fuite en avant qui ne permettra pas de résoudre la situation. D'autant que les expériences dans plusieurs pays ont révélé que le recours à la planche à billets a un effet pervers avec une augmentation des dépenses publiques. L'augmentation de la masse monétaire va engendrer une hyperinflation, faire perdre de la valeur à la monnaie nationale et rendre les importations, dont dépend le pays, beaucoup plus onéreuses».

\section{Conclusion :}

Un déficit budgétaire à un impact économique qui peut selon certains économistes, être un stimulant pour l'activité économique par l'intermédiaire d'une politique de relance selon les principes du keynésianisme, ou simplement être un moindre mal dans certaines situations de récession. Pour d'autres économistes, un déficit budgétaire est toujours le signe 
d'une mauvaise gestion des fonds publics et de l'argent du contribuable et à ce titre, il doit être évité.

Le financement de ce déficit par le mécanisme du crédit non conventionnel « l'émission de la monnaie par la banque centrale » peut servir au développement économique si les fonds sont bien placés et bien contrôlé comme titre d'exemple la planche à billets aux pays développés comme les Etats-Unis, qui ont imprimé une énorme somme d'argent au cours des dernières années, qui est entré dans des instruments financiers, ce qui augmente les prix des obligations et des stocks, cependant, se n'est pas le cas de notre économie, où l'argent est destiné à la consommation ce qui cause l'augmentation des prix.

Donc, il faut donc éviter de comparer l'incomparable comme le souligne Dr Mebtoul (Abderrahmane MEBTOUL, 2017) «Evitons de fonder une politique économique sur des modèles économétriques appliqués aux pays développés déconnectés des réalités nationales».

\section{Bibliographie :}

(s.d.). Récupéré sur http://.www.toupie.org/Dictionnaire/Politique_budgetaire.htm

Abderrahmane MEBTOUL, P. d. (2017, OCTOBRE 02). Financement non conventionnel : Comparaisons internationales et contradiction sur le montant de la sphère informelle.

Abderrahmane RAOUIA. ( 2017, Octobre 12). dificit budgetaire.

De Janvry. (2017, AVRIL 10). « Module 3 : les instruments de protection et leurs conséquences " Consulté le JANVIER 12, 2019, sur :http://www.fao.org/docrep/003/x7352f/x7352f03.htm

Dictionnaire d'économie et des sciences sociales. (1992). paris: Edition Nathan.

Gerard Thoris. (2000). « la politique budgétaire ». paris : caisse des dépôts et consignation, service de la recherche.

Lexique boursier. (s.d.). Récupéré sur: https://www.tradingsat.com/lexiqueboursier/definition-deficit-budgetaire-486.html

Loi n ${ }^{\circ}$ 17-10, r. à. (2003, août 26). Loi n ${ }^{\circ}$ 17-10 du 20 Moharram 1439 correspondant au 11 octobre 2017 complétant l'ordonnance $\mathrm{n}^{\circ}$ 03-11 du 27 Joumada Ethania 1424 correspondant au 26 août 2003 relative à la monnaie et au crédit. Loi $n^{\circ} 17-10 \mathrm{du}$ 20 Moharram 1439 correspondant au 11 octobre 2017 complétant l'ordonnance $\mathrm{n}^{\circ}$ 03-11.

Matthieu, L. (1998). « la politique budgétaire de la gestion de la demande », . PARIS: PUF-.

Saddiki, S. (Décembre 2014). « le trésor public », . Algérie.

Topictionnaire. (s.d.). dictionnaire la topie. Consulté le JANVIER 12, 2019, sur: http://www.toupie.org/Divers/recherche_resultat.php?mot=DEFICIT\%20BUDGE TAIRE

Tourev. P. ( 2015, octobre 27 ). Définition de la politique budgétaire», 27 octobre 2015, $\begin{array}{lllll}\text { Consulté le } & \text { JANVIER } & \text { 2019, }\end{array}$ http://.www.toupie.org/Dictionnaire/Politique_budgetaire.htm 